\title{
X-RAY STRESS ANALYSIS BY USE OF AN AREA DETECTOR
}

\author{
A. SCHUBERT, B. KÄMPFE and S. GOLDENBOGEN \\ Fraunhofer-Einrichtung für Zuverlässigkeit und Mikrointegration (IZM), \\ Gustav-Meyer-Allee 25, D-13355 Berlin, Germany
}

(Received 14 February 1995)

\begin{abstract}
The most commonly known techniques of $X$-ray residual stress analysis use diffractometers with conventional counters or position sensitive detectors. The $\sin ^{2} \psi$-method is often applied to $X$-ray stress determination, but requires various measurements at different sample positions. This technique was combined with an imaging plate, which is an X-ray digital area detector. The use of a two-dimensional detector like an imaging plate offers the possibility to get diffraction in one measurement from lattice planes lying on the whole diffraction cone. Thus the number of measurements for the calculation of the stress components can be significantly reduced. In addition, the use of an imaging plate has several advantages for the stress calculation even for materials having relatively large grains or a strong texture. Measurements on an electron beam hardened steel sample are described as an application example.
\end{abstract}

KEY WORDS: X-ray diffraction, residual stress analysis, imaging plate system, one-dimensional and two-dimensional approach, absorption and information depth, peak resolution, stress measurement on electron hardened steel.

\section{INTRODUCTION}

The X-ray diffraction method for stress measurements has been successfully carried out for many years (Hauk and Macherauch, 1984, for instance). The basis for the determination of residual and load stresses is the Debye-Scherrer-method and Bragg's law. The Bragg angles of one or several reflections are measured with high precision in the back reflection region. The results permit the determination of changes in interplanar spacings of polycrystalline materials.

Conventional stress analysis is based on two commonly used techniques, the so called " $\sin ^{2} \psi$ "- and " $\varphi$ "-methods (Macherauch and Müller, 1961; Kämpfe and Michel, 1986), which require measurements at different tilting and rotating positions of the sample with the help of a $\psi$ - or $\Omega$-goniometer (Hauk and Macherauch, 1984; Schubert $e$ t al., 1993; $a$ and $b$ ). In these cases the interplanar spacings and consequently the shift of the peak can be determined with conventional counters or position sensitive detectors. From the whole diffraction cone only a small part of lattice planes $\{h \mathbf{k l}\}$ with a special orientation $(\psi, \varphi)$ is analysed. The use of a two-dimensional detector like an imaging plate (STOE \& CIE GmbH, 1992) offers the possibility to get diffraction patterns in a single measurement from lattice planes \{hkl\} having one $\psi$ and all $\varphi$ orientations (non-tilted case) or different $\psi$ and different ( $\varphi$ orientations (tilted case). Thus, the number of measurements for the calculation of the stress components can be significantly reduced. Moreover, it is possible to investigate both textured materials and coarse-grained samples (Schubert et al., 1994, $a$ and $b$ ). 


\section{CONVENTIONAL METHOD OF X-RAY STRESS ANALYSIS (ONE- DIMENSIONAL APPROACH)}

In polycrystalline materials, the stress determination can be applied with the help of a one-dimensional detector if the grains are randomly oriented and sufficiently fine-grained so that the Debye-Scherrer cones consist of uniformly distributed diffractions spots. Under these conditions, it is possible to apply isotropic elasticity theory to convert determined strains into stresses. The residual strain $\varepsilon \varphi \psi$ can be determined from the change in lattice spacing $D \varphi \psi$

$$
\left(\varepsilon_{33}^{\prime}\right)_{\varphi \psi}=\left(D_{\varphi \psi}-D_{0}\right) / D_{0}
$$

where $D_{0}$ is the unstressed lattice spacing. This strain may be expressed in terms of the strains in $\varepsilon i j$ the sample coordinate system by the tensor transformation

$$
\begin{aligned}
\left(\varepsilon_{33}^{\prime}\right) & =g_{3 k} g_{31} \varepsilon_{\mathrm{kl}}=g_{31}^{2} \varepsilon_{11}+g_{32}^{2} \varepsilon_{22}+g_{33}^{2} \varepsilon_{33} \\
& +2 g_{31} g_{33} \varepsilon_{13}+2 g_{32} g_{33} \varepsilon_{23}+2 g_{31} g_{32} \varepsilon_{12}
\end{aligned}
$$

where $g_{3 \mathrm{k}}$ and $g_{31}$ are the direction cosines between $\mathrm{L}_{3}$ (measuring direction) and sample axes $S_{k}, S_{1}$ respectively (Hauk and Macherauch, 1984; Schubert et al., 1993, a). Primed tensor quantities refer to the laboratory system $\mathrm{L}_{\mathrm{i}}$, and unprimed tensor quantities refer to the sample coordinate system $S_{i}$. The direction cosine matrix for this case is

$$
g_{i k}=g_{\psi}{ }^{\mathrm{s} 2} g_{\varphi}^{\mathrm{s} 3}
$$

Since $\varepsilon_{\phi \psi}$ depends on the angles $\psi$ and $\varphi$, there are two basic measuring strategies the $\sin ^{2} \psi$ - and $\varphi$-method (Macherauch and Müller, 1961; Kämpfe and Michel, 1986). The measurement in various sample directions involves considerable time expediture and even the use of a position-sensitive detector can not always reduce the time taken to make such measurements:

- In the case of coarse-grained material only a small number of crystals is recorded by the one-dimensional detector. Then the assumption of isotropic elastic behaviour is no longer satisfied, since the reflecting single grains are elastically anisotropic. - Due to the elastic and plastic anisotropy resulting from texture, in most cases non-linear D vs. $\sin ^{2} \psi$ distributions or only a limited $D$ range of $\sin ^{2} \psi$ are observed. The measurement is selective in the sense that only those grains properly oriented to diffract contribute to the diffraction profile.

To overcome the described problems, an imaging plate would be helpful for stress analysis. Since the beginning of $\mathrm{X}$-ray diffraction, films have been used but they have some disadvantages because of the long time needed for picture development, film shrinkage, and the unsuitability of film data for direct computer processing. At last the film technqiues are now exchanged by modern two-dimensional detectors or imaging plates which allow the digitization of the whole diffraction pattern. 


\section{ADVANTAGES OF THE IMAGING PLATE}

Laser stimulated fluorescence imaging plates have been in use since 1986 as two-dimensional area detectors. The large dynamic range, high sensitivity, and low background render them ideally suitable as area detectors in X-ray crystallography (STOE \& CIE GmbH, 1992).

The experimental arrangement is shown in Figure 1.

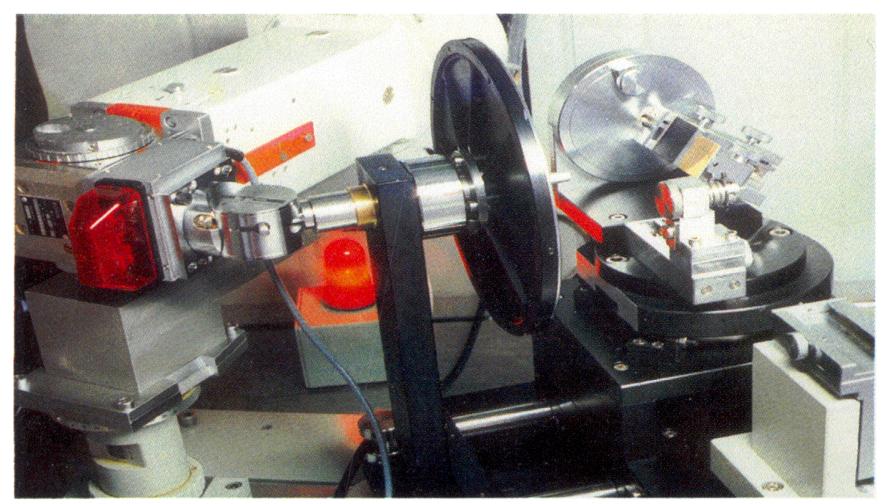

a)

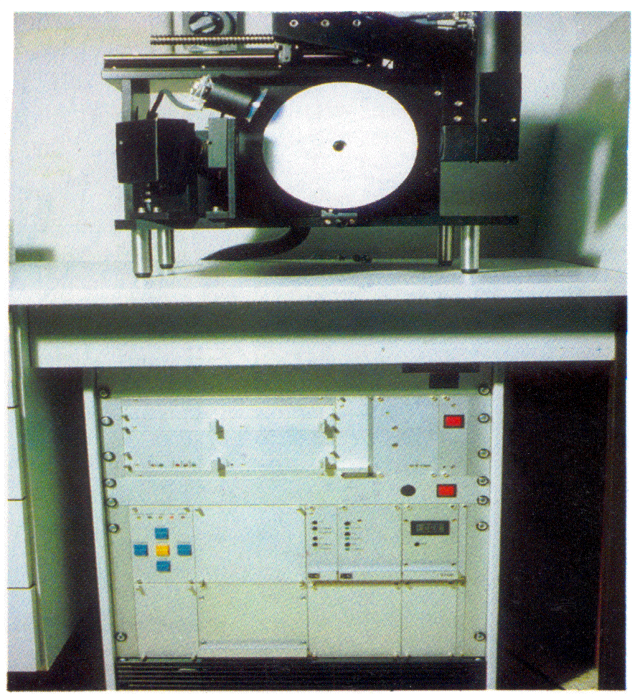

b)

Figure 1 Experimental arrangement (a) and read out (b) of the imaging plate (See Color Plate 1). 
The use of an imaging plate as a two-dimensional detector has several advantages for stress analysis

- fast and improved data processing

- better stress analysis for coarse-grained material by intensity integration of sectors or the whole Debye-Scherrer cone or by intensity measurement at discrete orientations

- improved stress determination for textured material by intensity measurement at preferred orientations (texture components), which are directly visible on the imaging plate (Figure 2)

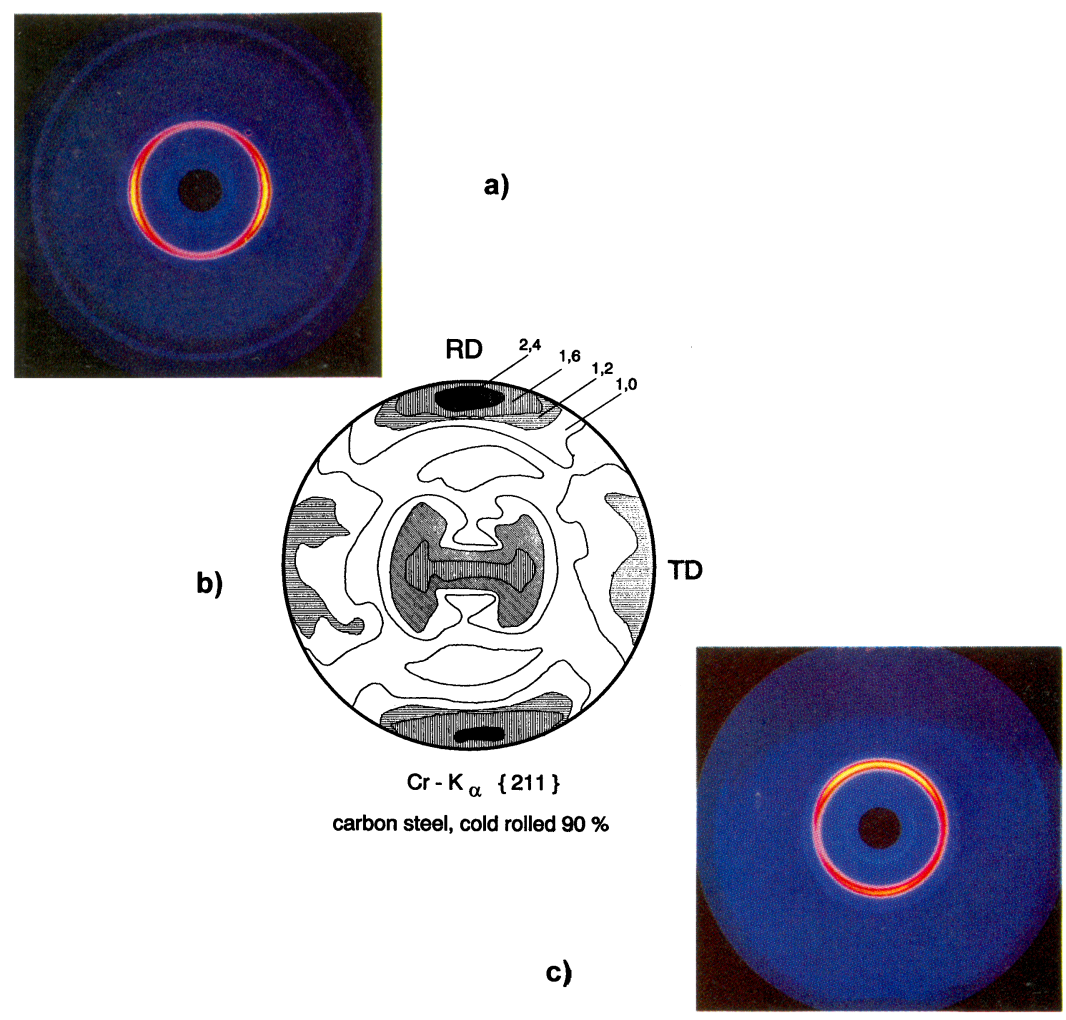

Figure 2 Intensity measurements at preferred orientations (texture components), which are directly visible on the imaging plate (cold rolled iron, $90 \%$, pole figure $\{211\}, \mathrm{Cr}-\mathrm{K} \alpha$ ), contour lines in $10^{-3}$ counts $s^{-1}$ a) image $\psi=0^{\circ}$, b) pole figure, c) image $\psi=60^{\circ}$ (Sec Color Plate 2$)$. 
- simultaneous determination of diffraction cones of different reflections in one measurement

- possibility of local stress analysis by combination of an imaging plate and X-ray microbeam diffraction technique

\section{TWO-DIMENSIONAL APPROACH (IMAGING PLATE)}

The incident X-ray beam is diffracted into a cone with an aperture angle of 2 $\left(180^{\circ}-2 \theta\right)$. An imaging plate perpendicular to the cone axis intercepts the cone in a circle if the specimen is unstressed (Figure 3). If stresses exist, an ellipse-like pattern occurs. The deviation of its shape from a circle is caused by stresses (strains), (Borgonovi and Gazzara, 1989; Schubert et al., 1994, $a$ and $b$; Yoshioka and Ohya, 1994).

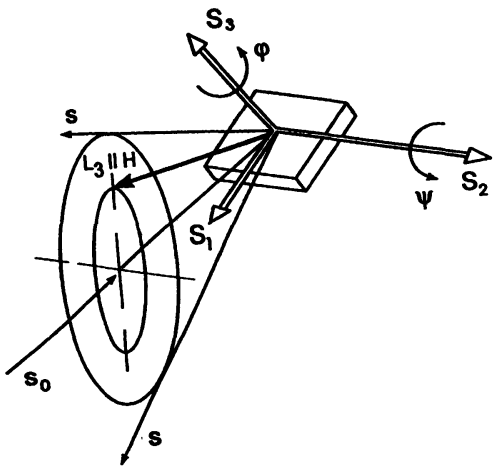

a)

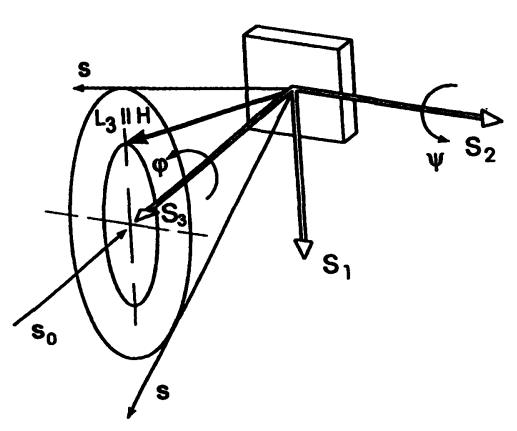

b)

Figure 3 Geometry of the two-dimensional approach (a) tilted and (b) non-tilted case

A point on the circle of the imaging plate corresponding to a diffracted beam can be found by the polar coordinates radius $R$ and the azimuthal angle $\beta$ with respect to the laboratory system. Once the position of the detector and the incident beam is known, it is easy to derive the angular position $2 \theta$ as well as the position of the diffraction peaks from the coordinates of the spot on the detector for various $\beta$ (Figure 4). All the normals of the crystal lattice planes responsible for the diffraction ring describe a cone with an aperture angle of $180^{\circ}-2 \theta$. A special normal is also characterized by the azimuthal angle $\beta$. In the sample coordinate system the same normal is described by the azimuthal angle $\varphi$ and tilt angle $\psi$.

To establish the correspondence between the sample orientation and the twodimensional detector, two coordinate systems are used. Figure 3 shows the relative orientation of the laboratory and sample system. The sample system is defined by the $S_{3}$-axis along the normal direction and the other two axes $S_{1}$ and $S_{2}$ in the surface. $L_{\mathrm{i}}$ is the laboratory system, with the $L_{3}$ axis oriented in the direction of the normals of the reflecting crystal planes. 


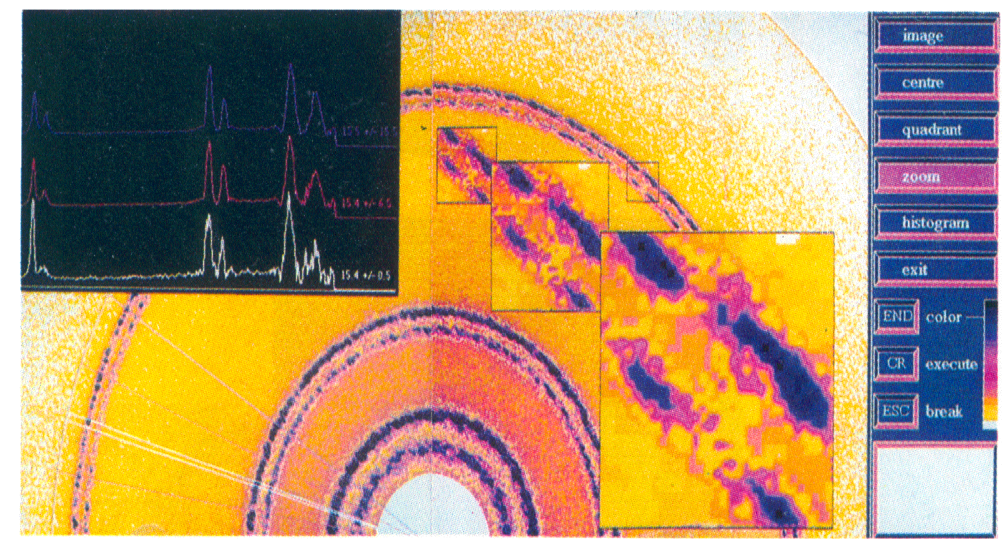

a)

b)
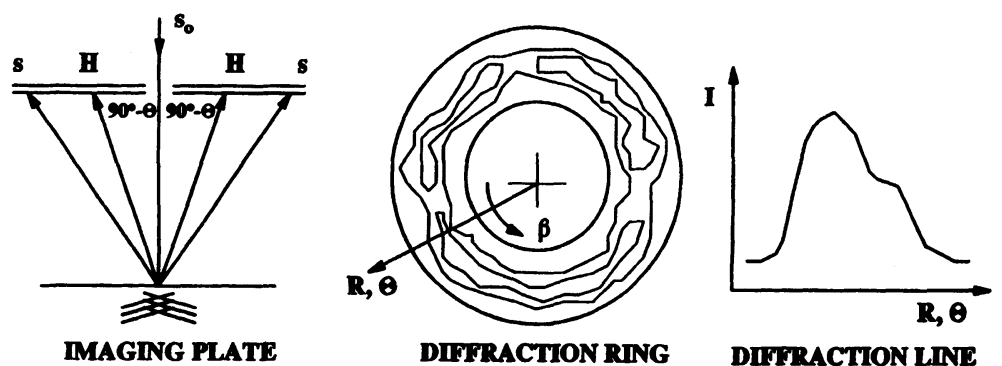

Figure 4 Sector analysis of the imaging plate (a) imaging plate and (b) principle. (See Color Plate 3).

Using the angles $90^{\circ}-\theta, \beta$, and $\psi$, the transformation of the sample system into the laboratory system is given by three consecutive rotations. The rotation matrix is of the form

$$
g_{\mathrm{ik}}=g_{90^{\circ}-\theta^{\mathrm{S}_{2}}} g_{\beta}^{\mathrm{S}_{3}} g_{\psi}^{\mathrm{S}_{2}}
$$

Now, substituting $g_{3 \mathrm{k}}$ and $g_{31}$ in (2), the fundamental equation of X-ray strain determination for the two-dimensional approach with an imaging plate is given. 


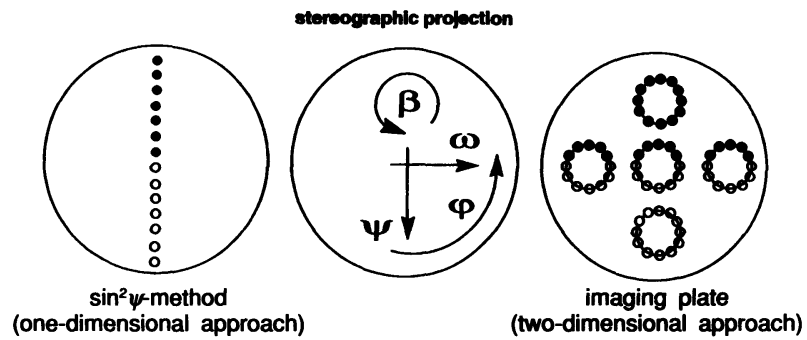

Figure 5 Representation of the one-dimensional $\left(\sin ^{2} \psi\right.$-method) and of the two-dimensional (imaging plate) approach by the help of stereographic projection

The difference between the one-dimensional approach $\left(\sin ^{2} \psi\right.$-method) and the two-dimensional approach (imaging plate) can be shown using stereographic projection. Figure 5 points out that for the same $\psi$-tilting the positions of the lattice spacings measured by the $\sin ^{2} \psi$-method do not correspond to the positions of the imaging plate.

\section{EXPERIMENTAL CONSIDERATIONS}

\subsection{Absorption}

A further point that must be considered in the analysis is the presence of strain gradients with depth. In such cases, the D spacings, and thus the strain obtained by the X-ray beam will be the average of this gradient over the effective penetration depth $\tau$ of X-ray.

The diffracted intensities are affected by absorption, which is directly related to the path length of the incident and diffracted beams in the specimen for a given geometry (Figures 6 and 7).

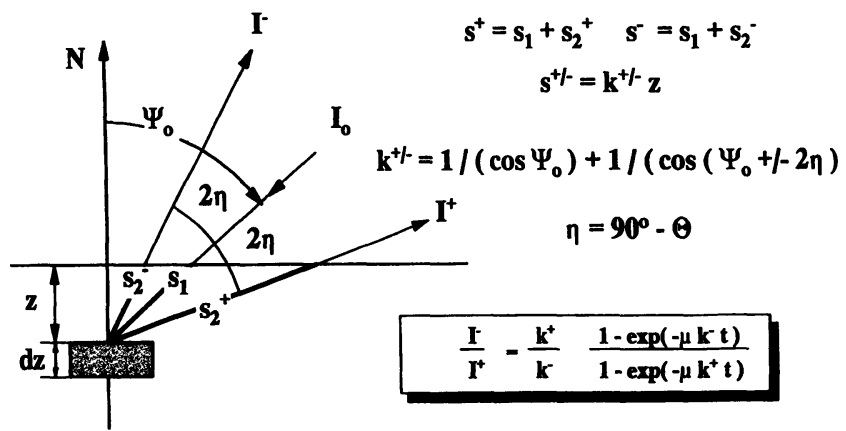

Figure 6 Geometry of the incident and diffracted beams (lower and upper position of the diffraction cone) 


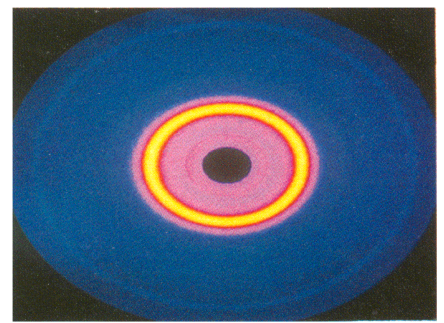

a)

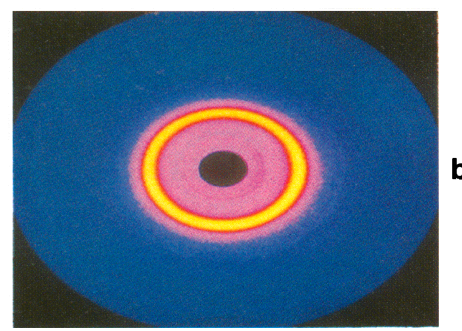

b)

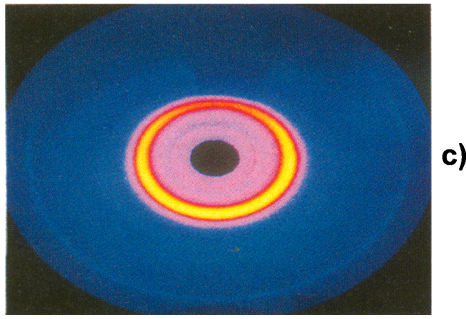

c)

Figure 7 Diffraction rings for different $\psi$-tiltings (iron, $\mathrm{Cr}-\mathrm{K} \alpha-\{211\}$ ) a) $\left.\psi=0^{\circ}, \mathrm{b}\right) \psi=24^{\circ}$, c) $\boldsymbol{\psi}=48^{\circ}$. (See Color Plate 4$)$.

Consider the case where a beam of intensity $l_{0}$ of unit cross section $F_{0}$ is incident on a flat plate at an angle $\psi_{0}$. The decrease of the intensity by a layer of thickness $d z$ at $a$ depth $z$ below the surface is described by the absorption factor (Schubert et al., 1993, $a$ and $b$ )

$$
A=\exp [-\mu k z]
$$

where $\mu$ denotes the linear absorption coefficient and $k$ is a trigonometric function determined by the diffraction geometry

$$
k^{ \pm}=1 /\left(\cos \psi_{0}\right)+1 /\left(\cos \psi_{0}+/-2 \eta\right)
$$




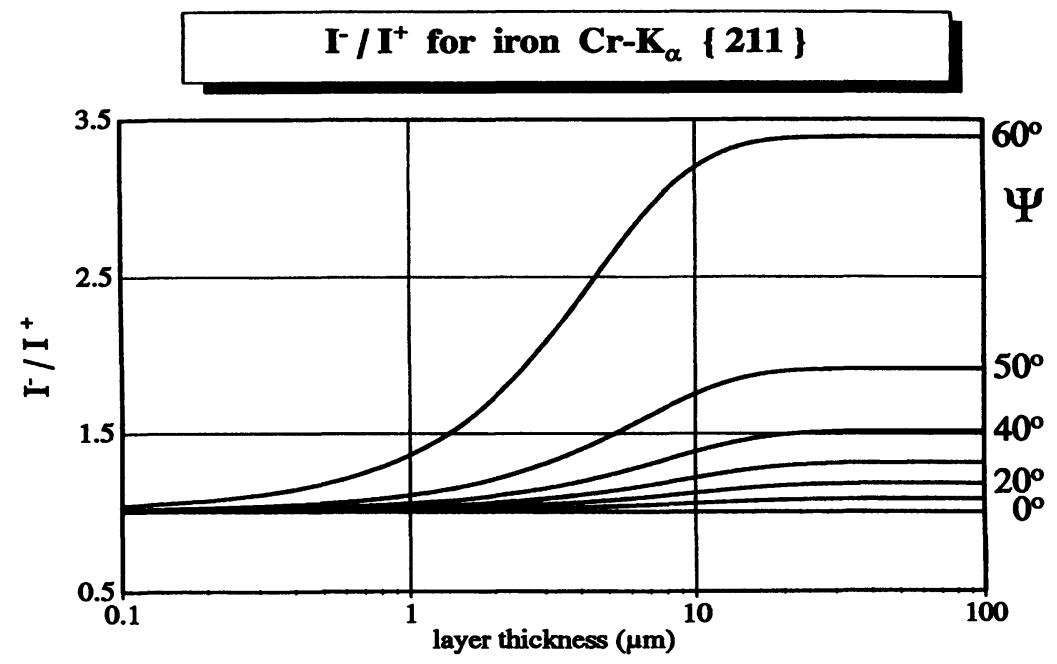

Figure 8 Intensity ratio of the lower and upper diffracted beams for different sample thicknesses and $\psi$-tiltings

with $\eta=90^{\circ}-\theta$ and $+/-$ for the upper and lower diffracted beam of the diffraction cone. The diffracted intensity is given by

$$
I=\int_{o}^{t} d l z=K \cdot B_{0} \cdot I_{0}\left(F_{0} / \cos \psi_{0}\right) \int_{o}^{t} \exp \left(-\mu z k^{ \pm}\right) d z
$$

( $K, B_{0}-$ two constants), (Figure 8).

\subsection{Exposure time and peak resolution}

Since the strains $\varepsilon_{\text {qu }}$ are less than 0,001 for elastic behaviour in most materials, the absolute value of the peak position, i.e. the peak maximum, is required for the accurate calculation of $D_{\text {ow }}$ from Bragg's law and an appropriate method of peak location must be used. The most important factor in X-ray stress measurements is that proper peak resolution must be achieved of all $\psi$ specimen orientation. The selection was based on obtained satisfactory fine-line peak resolution with X-ray intensities of at least $10^{3}$ to $10^{4}$ counts. Figure 9 shows $\{420\}$ reflections of a copper sample measured with $\mathrm{Cu}-\mathrm{K}_{\alpha}$ radiation. For data evaluation we reduce the two-dimensional image to a one-dimensional diffractogram for a given measuring time.

To determine the influence of the exposure time on the quality of measured Debye-Scherrer rings the imaging plate was exposed for several times. The results are shown in Figure 10. An optimum ratio between peak and background and a maximum intensity of about 1000 counts were determined for an exposure time of about $15 \mathrm{~min}$. For the $2 \theta$ peak location 3rd order curves were fitted through the data points using a least square fit. 


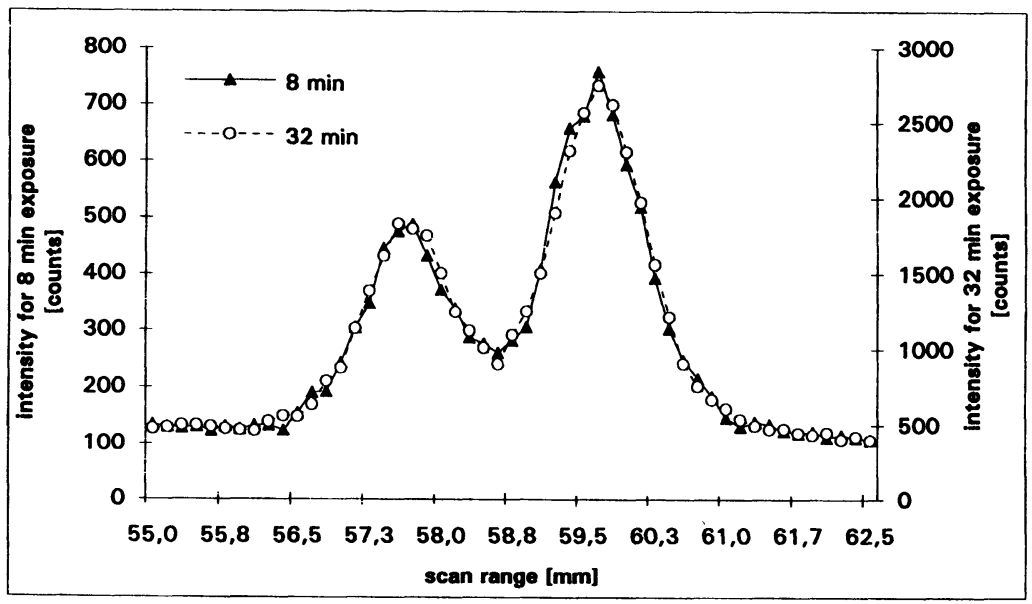

Figure $9\{420\}$-reflections of a copper sample measured with $\mathrm{Cu}-\mathrm{K} \alpha$ radiation for two exposure times (8 and 32 minutes)

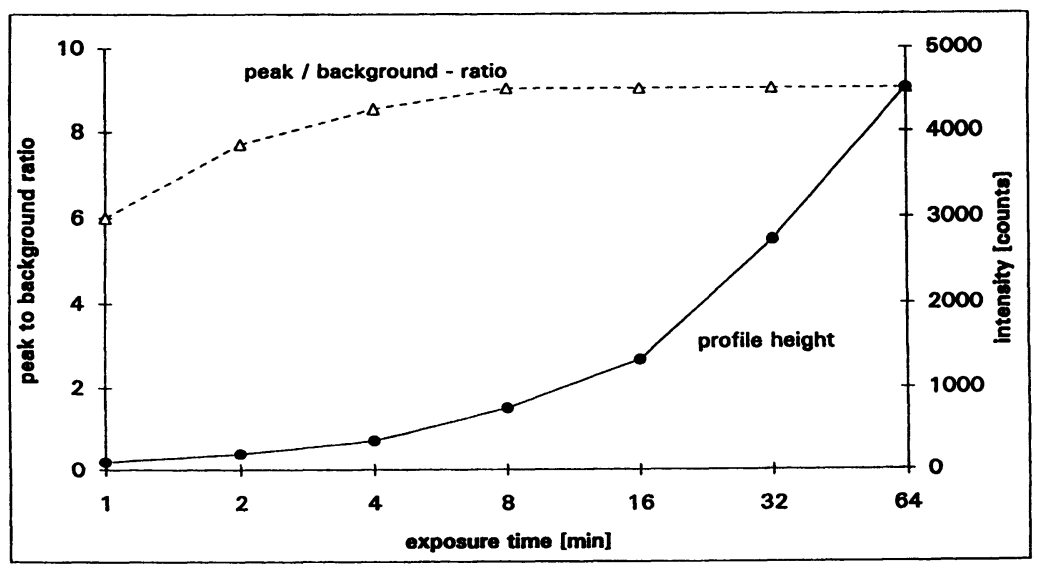

Figure 10 Ratio of peak to background and the maximum intensity for different exposure times

\section{FIRST EXPERIMENTAL RESULTS}

An example is given for using the two-dimensional approach to determine residual stresses in an electron beam hardened steel. The measurement was carried out using a conventional sealed tube $\left(\mathrm{Cr}-\mathrm{K}_{\alpha}\right.$-radiation, $\left.U=40 \mathrm{kV}, I=25 \mathrm{~mA}\right)$ and a graphite 


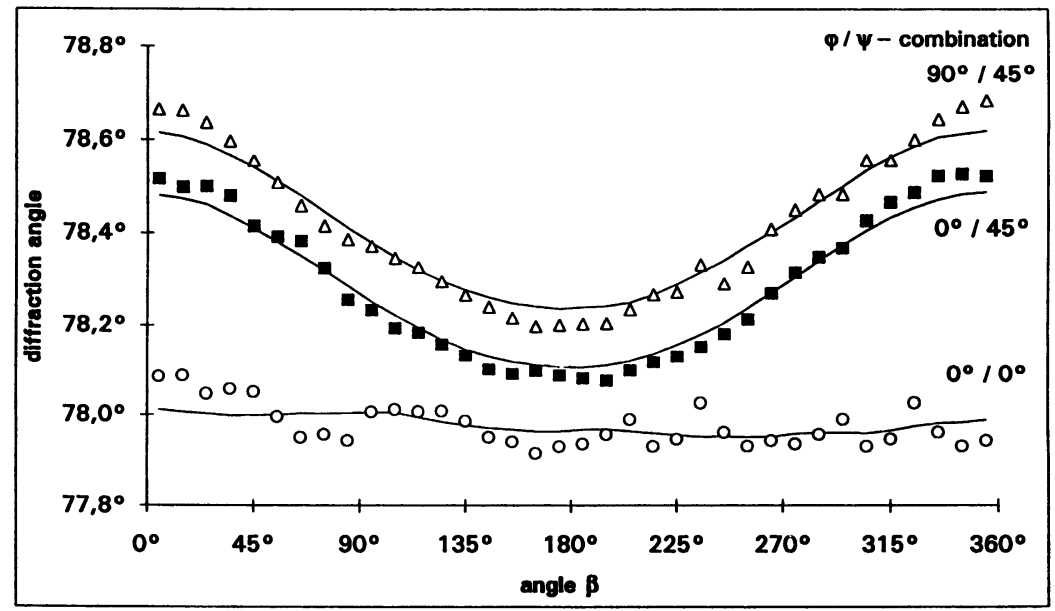

Figure 11 Diffraction angle vs. azimuthal angle $\beta$ for different $\varphi / \psi$-combination

monochromator. The distance between the imaging plate and the sample was $110 \mathrm{~mm}$ which corresponds to an observed region of $2 \theta=136,5^{\circ} \ldots 174^{\circ}(d=0,1233 \ldots 0,1147$ $\mathrm{nm})$. The collimator of $1 \mathrm{~mm}$ diameter is fixed in the hole of the imaging plate and guides the primary beam between the monochromator and the sample. The radius $\mathbf{R}_{s}$ of the peak position was determined for various azimuthal angles $\beta$ and ( $\varphi / \psi$-combination of $0^{\circ} / 0^{\circ}, 0^{\circ} / 45^{\circ}$, and $90^{\circ} / 45^{\circ}$ (Figure 11). The observed $R_{s}$ values were corrected using a standard powder which must correspond to a circle in contrast to an ellipse-like pattern caused by stresses. Then the $R_{s}$ were converted into $2 \theta_{s}$ values for the sample. Assuming the isotropic elasticity theory, the strain tensor $\varepsilon_{i j}$ was converted to the stress tensor $\sigma_{i j}$. The resulting stress components $\left(\sigma_{\text {imaging plate }}\right)$ are

$$
\begin{aligned}
& \sigma_{\text {imaging plate }}=\left(\begin{array}{llr}
-568 & -33 & 63 \\
& -624 & 8 \\
& & 0
\end{array}\right) \mathrm{MPa} \pm\left(\begin{array}{llr}
13 & 19 & 3 \\
& 14 & 3 \\
& & 15
\end{array}\right) \quad M P a \\
& \sigma_{\text {difraction }}=\left(\begin{array}{llr}
-388 & 24 & 16 \\
& -554 & 0 \\
& & 0
\end{array}\right) \mathrm{MPa} \pm\left(\begin{array}{llr}
25 & 15 & 6 \\
29 & & 6 \\
& & 22
\end{array}\right) \quad M P a
\end{aligned}
$$

The results are compared with the stress state determined with a conventional diffractometer $\left(\sigma_{\text {diffrection }}\right)$.

This two-dimensional approach looks very promising for the determination of residual stresses. For the electron beam hardened steel it was shown that this method - using an imaging plate - gave stress distributions which were in good agreement with those from stress measurements with a one-dimensional detector (Figure 12).

In the future, the absorption effects, the information depth and possible stress gradients have to be taken into account to improve accuracy of data evaluation. 


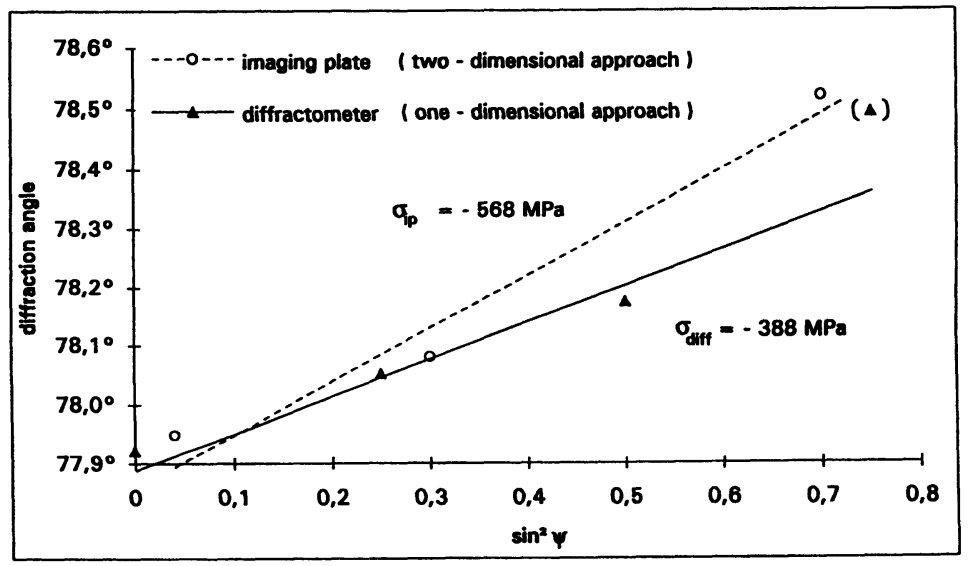

Figure 12: Comparison of the $\sin ^{2} \psi$-method in case of the two-dimensional (imaging plate) and of the one-dimensional (diffraction) approach

\section{References}

Borgonovi, G. M. and Gazzara, C. P. (1989). Stress measurements with a two-dimensional real-time system. Advances in X-ray analysis. 32, 397-406.

Hauk, V. M. and Macherauch, E. (1984). A useful guide for X-ray stress evaluation (XSE). Advances in X-ray analysis. 27, 81-99.

Kämpfe, B. and Michel, B. (1986). Diffraction analysis of stress states in surface layers. Acta technical Acad. Scie. Hung. 99, 313-315.

Macherauch, E. and Müller, P. (1961). Das $\sin ^{2} \psi$-Verfahren der röntgenographischen Spannungsmessung. Zeitschrift für angewandte Physik. 13, 305-312.

Schubert, A., Kämpfe, B. and Auerswald, E. (1993). X-ray analysis of residual stresses and textures in thin coatings. Materials Science Forum. 136-137, 117-122. Proc. EPDIC2. Enschede 1992.

Schubert, A., Kämpfe, B., Auerswald, E. and Michel, B. (1993). X-ray analysis of residual stress gradients and textures in thin coatings. In Residual Stresses, edited by V. Hauk, H. P. Hougardy, E. Macherauch, and H.-D. Tietz, pp. 663-671. Oberursel: DGM Verlag.

Schubert, A., Kämpfe, B., Ermrich, M., Auerswald, E. and Tränkner, K. (1994). Use of an X-ray imaging plate for stress analysis. Materials Science Forum. Proc. EPDIC-3. Vienna, 1993, in print.

Schubert, A., Kämpfe, B. and Michel, B. (1994). X-ray residual stress analysis in components of microsystem technology. Proceedings of the 4th International Conference on Residual Stresses. pp. 1113-1122. Baltimore.

STOE \& CIE GmbH (1992). Automatic X-ray powder diffractometer systems. 35-37.

Yoshioka, Y. and Ohya, S. (1994). X-ray measurement of residual stress in a localized area by use of imaging plate. Proceedings of the 4th International Conference on Residual Stresses. pp. 283286. Baltimore. 